\title{
Common Sense High Reliability Organizing (HRO) in the Response to COVID-19
}

Daved van Stralen, MD, FAAP, Thomas A. Mercer, RAdm, USN

\section{Abstract:}

We find descriptions of common sense in the domains of anthropology, artificial intelligence, and psychiatry. This is the common sense for adaptation, a form of practical intelligence that better predicts success in everyday experiences, if not real-world survival. This common sense is experienced-based knowledge rather than rule-based. When unexpected situations occur, it supports continuous assessments and decisions to adjust to the conditions at hand. Overwhelmingly practical, common-sense deals with a concrete situation on its own terms. Common sense describes cultural knowledge and behaviors. Inquiry drives practical common sense.

\section{"Proficiency in solving academic} problems appears to decline from early to late adulthood, while the ability to solve practical problems is maintained or increases through late adulthood (1). We respect academic prowess, yet we seek practical expertise."

\section{Introduction}

"We want someone experienced." Proficiency in solving academic problems appears to decline from early to late adulthood, while the ability to solve practical problems is maintained or increases through late adulthood (1). We respect academic prowess, yet we seek practical expertise.

The "old hand" had a sixth sense, always seeming to know where failure could occur. This was not a "devil's advocate" approach without the philosophical depth that criticizes without solutions, nor is it fear-mongering. The old hand pondered the situation and took straightforward action. Or told us to "do it." The authors recall these old hands who could foretell failure in the future. Just as often, they told us what to do when we could not see that future. It seemed needless work at the time, for something that we could not understand how it could happen. Journeymen, ironworkers, and miners always watched the novice $(2,3)$. Other times they watched us struggle, just far enough away, it seemed, to gloat at our hapless efforts getting the job done. The moment we struggled the most, the old hand walked over and gave us a simple solution, watching us, waiting with us for the moment we needed them. They were always teaching in this no-nonsense way, and it was after we had accumulated experience only $(1,4)$ that we recognized what we had learned from them. When events overwhelmed us, these same old hands walked up, saying, "Life's tough." They were in commiseration; we knew, at that moment, they had once been where we were then. All along, they had been helping us, standing along with us. They would never leave.

The authors learned this form of common-sense problem solving, also known as "lessons learned in blood," through similar dynamics starting decades apart in multiple, diverse domains. One author (DvS) queried experienced people in numerous countries and disciplines about their early, formative years. Did they have such old hands? It seems uniform; they all did.

\section{"Various definitions interfere with} discussions of common sense used by operators in high-risk situations. Our paper discusses common sense from intimate connection with the environment, knowledge, and experience handed down and focuses on consequences from inaction and consequences of actions taken."

Numerous disciplines discuss common sense as an entity: highrisk occupations (5), philosophy $(6,7)$, science (8), psychiatry (9), psychology $(1,10)$, anthropology $(11)$, sociology $(12,13)$, social psychology (14), logic (15), reasoning (16), artificial intelligence (17), and robotics (18). Various definitions interfere with discussions of common sense used by operators in high-risk situations. Our paper discusses common sense from intimate connection with the environment, knowledge, and experience handed down and focuses on consequences from inaction and consequences of actions taken.

This is the common sense for adaptation, a form of practical intelligence that better predicts success in everyday experiences, if not real-world survival $(1,19-21)$. This common sense is experiencedbased knowledge rather than rule-based (22). When unexpected situations occur, it supports continuous assessments and decisions to adjust to the conditions at hand.

\section{About Common Sense}

We do not accept the equivalency of common sense with common knowledge. Granted that common sense derives from shared, common knowledge, but our focus is solving the difficult problem embedded within the environment (23). This level of problemsolving is above that of the participant having acquaintance yet

NEONATOLOGY TODAY is interested in publishing manuscripts from Neonatologists, Fellows, NNPs and those involved in caring for neonates on case studies, research results, hospital news, meeting announcements, and other pertinent topics.

Please submit your manuscript to: LomaLindaPublishingCompany@gmail.com 
superficial experience with such situations. Working in direct danger with responsibility for self and others is quite different from standing alongside. We must recognize the situational and environmental effects on mental performance, awareness, reasoning, and leadership (24-27). Our discussion also does not include the superficial approach that relies on cliché, cool words, slang, and efforts to maintain the image of knowledge. Much of the academic criticism of common sense is directed at these categorizations of common sense. The visibility of practical common sense rapidly decreases with time and distance from events.

\section{"Much of the academic criticism of common sense is directed at these categorizations of common sense. The visibility of practical common sense rapidly decreases with time and distance from events."}

John McCarthy (15), in a position paper formalizing common sense for artificial intelligence, described common sense knowledge:

"Common-sense knowledge includes the basic facts about events (including actions) and their effects, facts about knowledge and how it is obtained, facts about beliefs and desires. It also includes the basic facts about material objects and their properties."

This is not common sense from the dominant account, generalized for standardization and support of the normative stance. Ours is the practical or operational common sense, a method absorbed in practice from voices without the privilege of being heard. Immersed in events hidden from spectators, this is knowledge too easily disregarded and lost.

Common sense has diverse meanings and uses. Common sense as shared beliefs and a shared way of thinking $(1,10)$ becomes embedded in social knowledge, binding a culture or social group to each other and their past. Sometimes referred to as "uncommon sense," what we generically refer to as common sense also describes methods of solving routine or everyday problems. While "old hands" often display common sense by helping novices assimilate into an organization, others weaponize common sense by isolating outgroups or individuals they judge as undesirable. Whether common or uncommon, such sense informs science even as it becomes part of the battle between scientific experts and laypeople, each perceiving themselves as having possession of expertise.

A pregnant mother with a highly infectious disease delivers an infant who does not have the infection. Management of the mother and infant follow published guidelines. The mother and infant are discharged without complications (28). Free of context, the reader could view this case report as trivial. The reader would then miss the influence of the pandemic COVID-19 as it brings together uncertainty, ambiguity, threat, stress, and fear, creating tumultuous medical, social, and political environments in which we operate that can, indirectly and unconsciously, drive treatment decisions (29).

The above case report focused on problem-solving through the structure of common sense rather than the use of common-sense knowledge. More specifically, this is solving problems from within using subjective common sense. Conversely, an objective com- mon knowledge perspective is associated with spectators or observers, a normative stance, or a fixed-point frame of reference (23). The spectator will not recognize "the thought and handwringing that has gone into making care plans for our newborns in the era of COVID. Some may even perceive this 'mindful indifference' as just 'indifference,' intellectual laziness, and/or lack of compassion/concern for the newborn," Poj Lysouvakin (29). Nor can the spectator appreciate the time and effort to gain common sense nor the anxiety during its application, described by Jaclyn Eisenberg(29):

The anxious feeling we all shared before walking into the room of a patient whose mother had COVID-19 and wondered if this particular patient could be the one to infect us while hoping that anxiety did not prevent us from providing good patient care. We also did not include the sense of helplessness we felt when we discharged this infant to a home where his mother was his primary caretaker - we did our best to keep him safe in the hospital but knew the best place for him was home with his family.

\section{"We describe this as the VUCA-2T environment (Volatility, Uncertainty, Complexity, Ambiguity-Threat, and Time Compression, see Table 1) (24), modified from the US Army concept VUCA $(33,34)$ and the anthropologic concept of liminality (35)."}

High-Reliability Organizing, an activity (the "verb" to organize for high reliability), engages the indeterminate, ambiguous, or illstructured problem embedded in the environment, addresses the abrupt, exigent circumstance, and responds to catastrophic failure $(23,30-32)$. We describe this as the VUCA-2T environment (Volatility, Uncertainty, Complexity, Ambiguity-Threat, and Time Compression, see Table 1) (24), modified from the US Army concept VUCA $(33,34)$ and the anthropologic concept of liminality (35). Such situations do not often readily translate into straightforward problems with definitive constituents, rules, and outcomes $(7,23$, 25). Inquiry is active and operational, supporting authority migration and information flow, enlarging small cues, and evaluating context $(7,32,36)$.

Table 1. VUCA-2T

\begin{tabular}{|l|l|}
\hline Volatility & The rapid, abrupt change in events \\
\hline Uncertainty & $\begin{array}{l}\text { Lack of precise knowledge, need for more } \\
\text { information, unavailability of the necessary } \\
\text { information }\end{array}$ \\
\hline Complexity & $\begin{array}{l}\text { A large number of interconnected, chang- } \\
\text { ing parts }\end{array}$ \\
\hline Ambiguity & $\begin{array}{l}\text { Multiple interpretations, causes, or out- } \\
\text { comes }\end{array}$ \\
\hline Threat & Impaired cognition and decision-making \\
\hline Time Compression & $\begin{array}{l}\text { Limitation acquiring information, deciding } \\
\text { or acting before consequential changes }\end{array}$ \\
\hline
\end{tabular}

Common Sense

Overwhelmingly practical, common-sense deals with a concrete situation on its own terms. The person "just knows it" $(11,37,38)$. 
"a person's thoughts and actions from an intelligent interpretation of a situation before acting $(1,15)$. The individual solves the problem not fully knowing which facts are relevant for problem-solving and obtaining knowledge about the environment solely through observation and participation $(15,37)$."

The common-sense world is one "in which we find things partly joined and partly disjoined" (39). Common sense is a method for practical problem solving, guiding a person's thoughts and actions from an intelligent interpretation of a situation before acting ( 1 , 15). The individual solves the problem not fully knowing which facts are relevant for problem-solving and obtaining knowledge about the environment solely through observation and participation $(15,37)$. An example is the experience of a senior school bus driver participating in evacuations preceding a hurricane landfall:

"A hurricane two days from landfall threatened nursing homes and trailer parks in a coastal city. School buses were the only vehicles that could accommodate and transport groups of people with their wheelchairs, bicycles, musical instruments, suitcases, and personal belongings. Except for wheelchairs, school bus drivers had no policy, procedures, or training for this, which included driving on the narrow roads of trailer parks with limited room to maneuver. Supervisors said drivers for the wheelchair buses were to follow FEMA instructions, not school board policy. The fire department provided each bus a firefighter responsible for what to do, where to go, and who could also monitor the rear of the bus for backing maneuvers."

"Several locations, especially nursing homes, had multiple school buses in a parking lot not meant for buses. There was no intuitive entry and exit drive for multiple buses. The only area in a city with sufficient space for maneuvering multiple school buses, with few exceptions, is the school bus yard. Bus drivers relied on a system with other trained drivers for backing and were unaccustomed to working with firefighters who used a different backing language. The personal belongings people brought did not strap down well using wheelchair straps."

"Arriving at a nursing home, I found buses in different directions, some drivers not sure which direction to drive. Some busses needed backing, but the drivers were not sure of the firefighters' backing directions. Difficulties loading several busses delayed their departure, further blocking movement. Knowing we were to follow FEMA instructions, I began shouting to the drivers, "We work for FEMA! So, we follow their rules!" [FEMA had no rules for this.] "Don't you know FEMA wants us to do this!" "We have to get this done!" Drivers moved their busses and figured out the backing instructions. I quickly developed a way to modify wheelchair straps to hold things. This was rushed due to demands from a large number of people."

"I arrived on a bus at another facility. A driver was directing traffic, but buses were arriving from both directions into the parking lot with inadequate space for turn-around and a single exit point where buses were also entering. All the drivers were earnestly arriving to remove patients. The dispatcher told the buses to use a specific entry, leaving about six buses on-site facing different directions. Using my previous phrases, I had one lane stop, and
I took a bus to drive it past the narrow bottleneck. When drivers saw their busses could fit, they followed. The buses could have blocked the only exit route had they become entrapped against each other while passing along the narrow drive. It would have been unclear which direction the buses should move to not increase the entrapment. This created a traffic flow pattern, the parking area emptied, and buses had room to enter."

"At another assisted care facility, the buses became jammed. I parked my bus out of the way. We had two drivers per bus. My partner helped to load wheelchairs while I directed traffic, again, using my "FEMA phrases." The buses arrived from multiple directions. I stepped in to develop a system for loading and directing traffic, then began directing buses to come from one direction. We had no plan for this, not even for a specific site. This continued throughout the day, constantly figuring out a plan at each site." Cary van Stralen, Pinellas County (Florida) School District

Although the use of common sense is deprecated from a normative frame or scientific view, we often define common sense by the absence of its use: a person who "lacks" common sense can appear hapless and easily confused as they blunder everyday problems; they have difficulty allotting attention to priorities, discerning relevance among facts; and they fail to comprehend their limited understanding. They do not understand what they do not understand $(11,15,38)$. Explaining things or teaching them can be difficult, perhaps due to "their" common sense being different or incompatible. When the person's lack of commo-sense places them in the "uneducable" category, it becomes a barrier from others or triggers self-selected departure.

We commonly describe the absence of common sense from the point of view of those who have common sense. The abrupt "loss" of common sense can occur to all of us, such as an abrupt loss of structure when learned rules do not work or the experience of being in a situation where we do not belong. We then become those hapless individuals sensing loss of control, impaired perception, failure of words, and distorted senses (19). This situational loss of common sense generates the feeling of vulnerability in the liminal zone (26) and is the basis for Karl Weick's (31) "loss of cosmology" to experienced people. It is appreciating the situational loss of common sense aids in modeling common sense for the novice and interpreting after-action reports. A patient without common sense due to pathology described the experience, "What is it that I am missing? It is something so small, but strange, it is something so important. It is impossible to live without it..." (40).

\section{"We commonly describe the absence of common sense from the point of view of those who have common sense. The} abrupt "loss" of common sense can occur to all of us, such as an abrupt loss of structure when learned rules do not work or the experience of being in a situation where we do not belong. We then become those hapless individuals sensing loss of control, impaired perception, failure of words, and distorted senses (19)." 
To assume common sense is lacking is to misread the reason common sense is missing or misunderstanding other causes that could contribute to the lack of common sense. Common sense is ecological; it has an adaptive purpose within a specific environment. A person acquires common sense as a learned, modeled behavior from an experienced senior individual within that environment. A person cannot acquire common sense without the opportunity to work with an experienced individual. Lack of common sense also occurs in those with intellectual limitations, acquired or congenital, and is a defining component for Autism Spectrum Disorders and schizophrenic vulnerability $(9,40,41)$.

More commonly, situational stress responses or fear reactions impair the cognitive abilities necessary for common sense (26). Organizational culture or leadership can sustain fear reactions that cause more damage to various processes than direct damage from the threat itself. This is the ecology of fear (42). How an organization processes information can also sustain fear responses. For example, a bureaucratic culture preoccupied with rules and positions or a pathological culture preoccupied with power and needs (43) creates the ecology of fear that impedes practical common sense.

\section{"How an organization processes information can also sustain fear responses. For example, a bureaucratic culture preoccupied with rules and positions or a pathological culture preoccupied with power and needs (43) creates the ecology of fear that impedes practical common sense."}

Conversely, "good" common sense is recognizable. We even admire its contributions to insightful problem-solving. Good common sense starts by effectively coping with everyday problems in an everyday way (11). Sensible people use all their senses for subtle and nuanced information from the environment. Judicious, intelligent, perceptive, reflective use of observation enhances their ability to apprehend the sheer actualities of experience. Common sense then leads to sensible conclusions (11). Common sense as

Table 2. Five characteristics of HRO an exquisite adaptation for complex or straightforward everyday problems brings perception and reflection to the issue $(11,38)$. This intelligence is practical rather than academic $(1,38)$.

We can discuss practical common sense for high-risk situations with five components - motivation, intention, knowledge, heuristic processes, and action. From these components emerges the five identified HRO characteristics (44) and the mechanisms to achieve HRO - sensemaking, and enactment (45). The reference for HRO activity is always to reality as experienced (4); reality drives action. HRO is practice, not theory. We must not treat these as categories or distinct entities; they operate through relations with each other.

Five components of common sense shared across diverse domains

Motivation - responsiveness to phenomena, specifically, pressure to correct disruptions and prevent failures.

Intention - to immediately act and adjust those actions, referencing an immediate, concrete goal.

Knowledge - tacit, concrete, background knowledge from experience that crosses domains.

Heuristic processes - intuition, insight, learning from experience (46).

Action - bridge gaps with engagement (23), think with motor cognition (4).

Inquiry drives practical common sense. For John Dewey (48), "Inquiry belongs to 'action or behavior,' which takes place in the world, not just within the mind or within consciousness." It is not uncommon for people to be introduced to HRO solely through the five HRO characteristics and their descriptions. As attitudes, the characteristics make plain the inquiry nature of HRO. While attitudes don't cause behaviors to the degree beliefs can, they do influence behavior. Through behavior, HRO "takes place in the world" as inquiry from common sense, a "natural attitude - from which humans see and experience the world" (9). The components of common sense, then, drive the HRO characteristics.

\section{Newborn and Tsunami Common Sense}

Common sense begins in the space between concreteness and the abstract, which can be described but not explained. The liminal character of common sense confounds translation to spectators and scientists, though they unknowingly rely on common sense. "Individual physical objects must be discovered before

\begin{tabular}{|l|l|l|l|}
\hline Characteristic & Description (44) & Attitude (47) & Behavior \\
\hline Preoccupation with Failure & $\begin{array}{l}\text { Lapse reflects a problem in the sys- } \\
\text { tem }\end{array}$ & Attitude toward failure & $\begin{array}{l}\text { Engage vulnerability or disrup- } \\
\text { tion }\end{array}$ \\
\hline Reluctance to Simplify & Reluctant to accept simplification & $\begin{array}{l}\text { Accept/seek complexity of } \\
\text { even simple events }\end{array}$ & $\begin{array}{l}\text { Respond to details, but appro- } \\
\text { priate details }\end{array}$ \\
\hline Sensitivity to Operations & Attentive to line work & $\begin{array}{l}\text { Awareness of how one fits } \\
\text { into the system }\end{array}$ & $\begin{array}{l}\text { Adjust for the disruption, but } \\
\text { continue routine operations }\end{array}$ \\
\hline Commitment to Resilience & Regain a dynamically stable state & Perseverance & Overcome failure in real-time \\
\hline Deference to Expertise & $\begin{array}{l}\text { Push decision making down and } \\
\text { around }\end{array}$ & $\begin{array}{l}\text { Respect the knowledge and } \\
\text { experience of others }\end{array}$ & $\begin{array}{l}\text { Create expertise by deferring to } \\
\text { expertise }\end{array}$ \\
\hline
\end{tabular}


abstractions can be made from their conceived nature," George Santayana (49). This chasm is the "gap between the apparent reality and one methodically ascertained; between phenomena in their concreteness and particularity, and facts stripped of all that is tangible and individual so that they may be fitted into some allembracing regularity" (50).

Mouth-to-mouth resuscitation of the newborn provides an example of delayed acceptance despite its use by medical professionals:

"In the country, a young woman was taken up and committed to jail to take her to trial for the supposed murder of her bastard child. According to the information which he had received, he was inclined to believe, from the circumstances, that she was innocent; and yet, understanding that the minds of the people in that part of the country were much exasperated against her, by the popular cry of a cruel and unnatural murder, he feared, though innocent, she might fall victim to prejudice and blind zeal.

"[H]aving concealed her pregnancy [concealment of pregnancy was a crime], the baby was delivered during the night by herself. She was suspected, and the room was searched, and the child was found in her box, wrapped up in wet clothes. She confessed that the child was her's but denied having murdered it or having had an intention to do so...[on autopsy] the lungs would not sink in water [a presumptive forensic sign of live birth]. It is so generally known that a child, born dead, may be brought to life by inflating its lungs, that the mother herself, or some other person, might have tried the experiment" (51).

Case I. "The lady of an officer was delivered of a stillborn child. The feet had presented, the labour had been quick, and there was reason to believe that life had not been long extinct. The lungs of the child were, therefore, immediately inflated from my own, while the body was immersed in hot water, and volatile spirit occasionally applied to the nose, mouth, and chest" (52).

Case II. "The wife of [a] housepainter, \&c. in Northampton, was delivered of her first child. The labour had been tedious, the liquor amnii having been discharged very early and the face having presented. The child, a female, was stillborn. The circulation of the funis had quite ceased, and the heart of the infant was perfectly still. The face had suffered from hard pressure, was swollen, and marked to a considerable extent with ecchymosis; the lips were a good deal enlarged, remarkably flabby, and pale. There was no sign of animation in any part of the body, and I thought a very faint hope of restoration. But, encouraged by the recollection of the preceding case, I determined on making an attempt, and the fortunate result of it, I have now the pleasure to communicate. Interposing a piece of muslin inflated the lungs from my own mouth, closing the nostrils by the pressure of my fingers. The thorax was compressed after each inflation, and thus artificial respiration was maintained, observing the natural periods of frequency, and keeping in mind the difference of capacity between the child's lungs and my own. In about half an hour, I felt very clearly a faint pulsation of the heart; a little fluttering, I thought I had perceived, once or twice at intervals, a few minutes before, but I was hardly sure of it" (52).

Case III. "After a very severe natural labour of 12 hours, the head was expelled, and the child was then alive. Although the expulsive uterine efforts continued, the body very unaccountably made no progress. The head had been protruded about a quarter of an hour when the child gave several convulsive jerks, indicating its being in danger. I endeavoured to get my finger into the armpit to bring the body down, but it was so high and the head so closely drawn to the external parts that I could not reach the armpit so as to act. After several efforts, the finger was at length hooked with difficulty round the arm, and the body brought down. A quarter of an hour was spent in extracting the body. This child was lifeless, and its appearance in every respect resembled the two former [lifeless at birth, successfully resuscitated]. No time was lost in breathing into the lungs and using the other means, and in half an hour, it gave the usual convulsive gasp to respire. The artificial inflation was carefully continued, and in seven minutes more, it made a second attempt. No progress was made in the respiration. The lungs were now very frequently inflated, and in a short time, the respiration began to improve, and for a minute or two would continue pretty regular, but, unfortunately, whenever the artificial inflation was given up, there was an evident falling off and a tendency to return to a state of stillness. It was not till after the expiry of three hours and a half from the birth that I ventured to desist, the natural respiration being then almost perfect. Everything continued to do well, and he became a very fine healthy boy."

"In estimating the comparative value of the different means used in these cases, I must give a decided preference to breathing into the lungs. No instruments were used; these I have found very troublesome, and I have never been able to inflate the lungs so completely by them as by simply applying the mouth to the child's, at the same time closing its nostrils and gently pressing back the trachea...In some of the cases gentle, and in others more powerful inflation, was found most efficacious."

"I am aware this practice has been objected to. It has been urged that inflating the lungs with air so charged with carbonic acid gas is more likely to destroy than restore life. This objection is plausible but cannot possibly be true. Were it so, no case, such as those detailed where this method was used, could have been restored. A thing of which the uniform tendency is to destroy life, never can in a single instance reanimate, so that these cases show most satisfactorily that expired air may be taken not only safely, but salubriously, into the lungs of another...[B]ut I am sure the usual method of blowing into the lungs does not merit that censure which has been cast upon it. While I would place my chief reliance on insufflation, persevering to repeat it at short intervals" (53).

Desperate young mothers gave mouth-to-mouth resuscitation to save their newborn babies. For these mothers, it was common sense. For physicians, elevated expired $\mathrm{CO}_{2}$ was "more likely to destroy than restore life." For the courts, the results became forensic evidence in the determination of infanticide or feticide, air in the lung evidence of infanticide. William Hunter described this in 1783 but as a problem for the mother. If the baby dies, then air in the lung suggests the baby was born alive. The vignettes describe how several physicians successfully administered mouthto-mouth resuscitation to newborn babies in the years 1814, 1817, and 1824, respectively. Such common sense lay dormant for over 150 years.

"William Hunter described this in 1783 but as a problem for the mother. If the baby dies, then air in the lung suggests the baby was born alive. The vignettes describe how several physicians successfully administered mouth-tomouth resuscitation to newborn babies in the years 1814, 1817, and 1824, respectively. Such common sense lay dormant for over 150 years." 
The concern for resuscitation about elevated $\mathrm{CO}_{2}$ in expired air continued through the 1940s. What is often missed to this date is mouth-to-mouth resuscitation to model bag-valve-mask (BVM) resuscitation. BVM is associated with barotrauma and gastric insufflation $(54,55)$, complications rarely observed with mouthto-mouth resuscitation. During mouth-to-mouth resuscitation, the rescuer feels the pressure change in lung compliance on inspiration and the end of expiration by the cheek. The author's (DvS) experience administering mouth-to-mouth breathing in the field to an infant, adolescent, and adult later informed his approach for BVM and mechanical ventilation for spontaneously breathing patients (56). As noted by Dr. Wilson in 1829, "I am aware this practice has been objected to."

"What is often missed to this date is mouth-to-mouth resuscitation to model bag-valve-mask (BVM) resuscitation. $B V M$ is associated with barotrauma and gastric insufflation $(54,55)$, complications rarely observed with mouth-to-mouth resuscitation. During mouth-to-mouth resuscitation, the rescuer feels the pressure change in lung compliance on inspiration and the end of expiration by the cheek."

Perhaps the difference in common-sense problem solving between the mothers and physicians is the personal impact. For example, a tsunami survival solution is to move to higher ground rapidly. Three tribal groups who survived the December 2004 Indian Ocean tsunami have lived in the region for between 30,000 and 50,000 years: the Moken of the Andaman Sea (57), the Onge tribe on Little Andaman, and the Jarawa on South and Middle Andaman Islands (58). Thousands of Nicobarese who migrated from South East Asia 500 or 600 years ago were dead or missing (58).

They had heard stories of laboon [the "seventh wave"] around the fire; when it comes, they had been told, go to the mountains or head to deep water. For the animist Moken, laboon was sent by ancestor spirits to clear out the world's evils. It would devour everything in its path before all was reborn. "A long time ago, there was a wave so big it covered all but the highest mountain," Tad, a Surin elder, told his family (57).

[The Onge tribe] folklore talks of "huge shaking of ground followed by a high wall of water," according to Manish Chandi, an environmental protection worker who has studied the tribes and spoke to some Onges after the disaster. The aboriginals know about tsunamis, and they know how to deal with them (58).

\section{The Practice of Common Sense}

We find descriptions of common sense in the domains of anthropology, artificial intelligence, and psychiatry. In anthropology, common sense describes cultural knowledge and behaviors (11, 50). Artificial intelligence struggles with several gaps that describe common sense - processing large amounts of information (17), the frame problem (37), and constraints due to logic (59). Psychiatry described common sense by its absence. In the early $20^{\text {th }}$ Century, the loss of common sense was a defining characteristic of autism and schizophrenia (41). The following is a distillation of the literature describing how common sense is used by practitioners.

\section{Psychosocial}

Common sense at the personal level preserves mental health and gives a sense of agency (40). Wolfgang Blankenburg, a German psychiatrist, conducted extensive studies on schizophrenia. He observed that, by overlooking the "obvious as obvious," we "resist" loss of our common sense, preserving our mental health. That is, the obvious does not require exploration (40), allowing us to see things in a fuller, proper perspective. We can quickly grasp the significance of life's discrepancies and disruptions, becoming attuned and grounded (60). Motor cognition comes from the coupling of perception and action, which is also the mechanism of common sense. The parietal cortex during motor cognition distinguishes oneself from others, an essential aspect of agency (61) that is lost in schizophrenia (40).

Common sense, as social knowledge, is the background knowledge for expectations, meaning, and the "rules of the game." It gives the ability to spontaneously share in activities with others $(19,40,60)$. Contextual understanding and motor cognition increase the capacity for attunement, the ability to appreciate and be involved with the mental states of others.

"Common sense, as social knowledge, is the background knowledge for expectations, meaning, and the "rules of the game." It gives the ability to spontaneously share in activities with others (19, 40, 60). Contextual understanding and motor cognition increase the capacity for attunement, the ability to appreciate and be involved with the mental states of others"

\section{Reasoning}

Common sense is improvable and adaptable. A person with common sense learns from new information. Though this appears trite, a computer program will evaluate if new information is relevant, conform to existing information, use the deduction for solutions, and not change a derived solution (the property of monotonicity) $(15,59)$. On the other hand, a person using common sense can use partially relevant information, distill experience, and a vast store of knowledge into an accessible representation when needed $(15,62)$. "We shall therefore say that a program has common sense if it automatically deduces for itself a sufficiently wide class of immediate consequences of anything it is told and what it already knows," John McCarthy (15).

Common sense draws its authority from reality by presenting the world in an accessible form, allowing us to derive information from the world $(11,62)$. Science gives us an explanation of reality, stripped of what is tangible and specific, creating a generalizable abstraction. Common sense then establishes cause-and-effect that is practical rather than theoretical, creating an image of reality for survival (19). Through concrete descriptions of reality, common sense bridges the gap between description and explanation (50). Common sense stimulates our initial approach, guides us at 
the beginning, and shows us the first solutions (50).

A person entering a new situation uses common sense to select memory structures, what matters from what does not matter, what is relevant or irrelevant, and selectively attends to the relevant, then changes the details as necessary $(11,59,60)$. For artificial intelligence, common sense must become larger and more intimately connected to the world with context formalized into objects $(15,59)$. Common sense accepts a sharp distinction between description, what we have in context, and explanation, what led to the context. Common sense can move forward from single facts because ordering a few facts is less of a problem. The person uses the senses judiciously, intelligently, perceptively, reflectively. Science does not make such a clear distinction between description and explanation. The context will complicate something new because ordering multiple facts is more complicated, so the next steps become less clear. The person with common sense copes with everyday problems, the foundation of "everyday" experience $(11,50)$.

Common sense arises from the distilled actualities of experience. In turn, immediate experiences interpreted through common sense feel more real $(11,19,62)$. Common sense is an attitude from which we experience the world, denying that it as is a body of knowledge while affirming that it comes without reflection from that experience $(9,11)$. If the experience does not fit common sense, then the character of the experience is ruled out (19). Lack of common sense in artificial intelligence makes the system "brittle," difficult to extend into new situations (63).

With common sense, we can distinguish relevant, irrelevant, or improbable (9) as well as information that is partially relevant or contingently relevant. Common sense does not have well-demarcated boundaries or compartmentalized knowledge (15). While more elementary than determining true or false, the ability to cross boundaries, use loosely coupled knowledge domains, and weigh value becomes vital in the world of experience. Without common sense, a reaction to this messy organization of knowledge is to demand certitude, construct controlled effortful processing, and have inflexible attachment to the 'axioms of everyday life' $(40,64)$.

\section{Problem Solving}

Common sense judges the probable abilities, causality, and the possible results of different actions instead of the truth $(37,40)$. The common-sense individual does not know in advance what actions to take. Therefore, goals are judged by the possibility of sequences that can reach the goal (37).

Using common sense, a person can change plans as information is added or updated. Deductive reasoning systems have the property of monotonicity, the term used for its analogy to similar mathematical concepts. In monotonic reasoning, new information does not change a valid conclusion. Adding assumptions for relevancy or reaching a specific conclusion only increases the number of conclusions without discarding the old conclusions $(59,63)$. Humans do not reason that way. When we receive new information, we adjust our conclusions. We reason non-monotonically because classical logic and scientific reasoning fail us in high-risk situations (65). Formalized modes of nonmonotonic reasoning added

Readers can also follow NEONATOLOG via our Twitter Feed @NEO to computer systems give "rules of conjecture" rather than "rules of inference." Conclusions appropriate in one set of assumptions can be disconfirmed by adding new assumptions (63). Nonmonotonicity brings artificial intelligence closer to common sense.

Common sense contains heuristic processes such as analogies. Paradoxically, the uncertain methods of partial matching through analogies are more effective at reaching conclusions than the certainty of logic. You do not need a new frame or analogy for every problem. Logic does not represent approximations for solutions, and "a number cannot reflect the considerations that formed it" (59). Science attempts to classify an observed object, subsuming the observation within a known object, thereby preserving order. On the other hand, common sense finds an analogy between what is explored and what is known. Though common sense starts with deductive explanations, it concludes with an analogy (50).

\section{"Novelty can distract as the person describes what is familiar or personally salient rather than used an analogy to develop relevance and meaning. Analogies can act to create a topological space for safer and more effective engagement."}

Analogies are used to understand and predict new features of novel domains. Common relations early in the experience are more important than common object descriptions (66). Novelty can distract as the person describes what is familiar or personally salient rather than used an analogy to develop relevance and meaning. Analogies can act to create a topological space for safer and more effective engagement.

\section{Topology}

We can understand common sense as a topological space where elements maintain continuity of connectedness despite deformations. The focus is on how the elements are connected, for example, the closeness of connection or overlapping connection (67). This topology differs from the academic approach forming knowledge into logical categories and scientific theories representing compartmentalized knowledge $(15,38)$. This logic initially seems counter-intuitive, but that is likely from exposure to three-dimensional Euclidean space that comprises much science education. Points and measures comprise Euclidean space, whereas connection and relation comprise the topological space.

Common sense deals with only partially known elements or with information that cannot be separated from irrelevant information (15). Quantitative measures, hierarchy, metrics as points and lines, or discrete representations do not work in the topological, common-sense space (59). Common sense organizes knowledge in topological forms connected for their value to solve problems and support social organization. For Norwegian fishermen, specific elements and categories of the fishing experience form systems and shared conceptions comprising common sense and meaningful social reality (5).

Facts will come together in some conjunction; if their relations are "right," then we have an explanation (50). If we have expectations, then we are surprised if the conjunction of facts has different relations. A topological space allows us to engage from observed relations. Topological space accommodates larger yet more intimate connections for common sense, giving more power and speed to 
mental activities (59).

Commonsense experiences time as continuous and branching. We can record the arrested event, elements at the moment in time and use the information to study and discuss. Within the event, the individual works with simultaneous events with the trajectory of the larger event. Though prediction has some value, it is the engagement of the situation where we use common sense to change the trajectory. This is the "sensed" event of experience that we cannot record. Euclidean space has categories; topological space does as well, but they are more open and overlapping with some elements in residual categories.

The topological space contributes to some of the difficulties individuals have described in how they use common sense. We do not have a natural way to describe points without measure. Spatial relations are privileged over relations and connected continuity (67). It seems paradoxical that a person or element at a greater distance influences a person than immediate events or an immediately adjacent colleague.

\section{"It seems paradoxical that a person or element at a greater distance influences a person than immediate events or an immediately adjacent colleague."}

\section{The Frame Problem}

Healthcare organizations have routine and contingency plans. Medical care units have patient care protocols. Robots have artificial intelligence programs. John McCarthy and Patrick Hayes (37) identified a problem due to the operational environment: "One assumes, as a rule of procedure (or perhaps as a rule of inference), that when actions are performed, all propositional fluents which applied to the previous situation also apply to the new situation." This is the frame problem - how to represent the effects of action without also representing all non-effects. If we add assumptions for the non-effects, monotonic reasoning describes the explosion of new conclusions without change in the original conclusions (59, 63). The frame problem is not part of planning or protocols.

Since its first identification, we now understand that the frame problem includes what properties change and what properties do not change. Any action in the NICU can affect unrelated but concurrent actions, complex interactions, and indirect actions. For example, when a physician responding to low $\mathrm{CO}_{2}$ levels decreased the ventilator rate and tidal volume for an adolescent on long-term ventilation, a respiratory care practitioner (RCP) reminded the author (DvS) of the reasons for the settings - frequent pneumonia. Also, a recent decrease in tidal volume due to low $\mathrm{CO}_{2}$ levels had resulted in hypoactive delirium, loss of awareness, and no smiling. At that time, after returning to the previous settings, the adolescent immediately opened his eyes and smiled (in the presence of the author). The problem-solving frame for the physician was mechanical ventilation and blood gas analysis. This situation can produce "euboxia," the belief that things are settled if "all the boxes have good values" (47).

Marvin Minsky (59) proposed that the primary purpose of problem-solving is to understand the problem space. Rather than search for a solution, we find representations or frames that make the problem easier to solve. In effect, rather than narrowing the search through simplification, we search for information that increases the complexity of the problem. Once we better understand the problem space, we more easily find a solution. This paradox is "reluctance to simplify" transformed to "willingness to complexify," possibly a more helpful characterization (Karl Weick, personal communication).

The frame problem contributes to, if not generates, errors. If a system cannot learn from errors from the frame problem, the errors will continue. This does not represent human reactivity (59). The change in the frame or its size interferes with the ability to foresee every consequence of an action. To do this, we would deduce the implications of actions from their descriptions in the plans using relevant implications while ignoring the irrelevant ones (68). Then, during operations, we swiftly gather information, particularly in the unexpected or where we are surprised. However, to be surprised, we would have expected something else, which takes much information. We can reduce our information load using stereotypes, which makes sense as there can only be so many combinations $(59,68)$. Nevertheless, stereotypes bring bias and prejudice. Common sense is a means of dealing with a large amount of relevant, partially relevant, conditionally relevant, and irrelevant information.

Another cause of frame problem error is the assumption that the representations of the environment accurately encode what it represents. These representations have some sort of correspondence to the actual world, for example, causal, informational, or structural. These representations can be correct, incorrect, or non-existent. We cannot calibrate our representations or mental models against the actual world. Cognitive models don't help us because the mental models in cognition are the sources of the frame problems (69).

\section{Common Knowledge, Tacit Knowledge}

We distinguish common sense as problem-solving from common sense as knowledge. Common sense and common knowledge are frameworks and phenomena which underwrite much of social life, holding us together as a social group $(8,70)$. Everyone in the social group knows common knowledge, AND all members know that other members have this knowledge as well. Common sense knowledge is the knowledge that ordinary people use in everyday situations focused on the practical and results rather than the structured, theoretical knowledge science uses to expand knowledge (38). Rather than laws dictating causality, common sense considers "influences," assuming "that almost everything is significant and can exert influence of some sort" (39).

The use of proverbs, jokes, and anecdotes rather than formal doctrines and theories (11) can appear trivial and inconsequential, misleading the spectator or outsider. Taking lightly, this form of common sense comes at grave risk. Compared to their use for general conversation, in a high-risk situation, such phrases intimately tie danger and consequences from an indistinct threat to concrete observations and actions. Common sense will then quickly elaborate a wide range of immediate consequences that artificial intelligence has difficulty doing (15). Common sense supports immediate action to avoid such hidden consequences, thereby producing immediate results. But the results are of a failure avoided. This context is an immediate enacted experience rather than deliberate reflection (11).

We may assume a lack of common sense when the difficulty lies in the assumption of mutual knowledge as common knowledge. Mutual knowledge is known by all, except that people don't know that everyone has that knowledge. During a period of multiple deaths and organ transplants, the fatigued PICU team described the transfer of a series of patients to the PICU service for brain death evaluation as "dumps." [A "dump" in healthcare refers to the transfer of care to another service to manage an "easily managed" problem.] The team defined it for the author (DvS) as the transfer of an easily managed patient to avoid work. The author described the admission of an infant, otherwise healthy but for an elective 
procedure, to a specialty surgical service in the PICU. This was early in the first year of the unit's existence. Would it have been a "dump" for the surgical service to ask the pediatricians to write feeding orders for the formula? The PICU team believed the surgical resident could contact parents or use house formula. The infant received a substantial volume of formula in relation to medications, aspirated, and then died. The team appreciated that what they had called a dump was simply a problem of common sense to them because of their knowledge and experience. The author never again heard the term "dump."

"The PICU team believed the surgical resident could contact parents or use house formula. The infant received a substantial volume of formula in relation to medications, aspirated, and then died. The team appreciated that what they had called a dump was simply a problem of common sense to them because of their knowledge and experience."

We can also look at how conclusions different from our own are reached (25). Even with error, "thought operates according to the model of common sense" (71). Discovering an error, the organization would turn to remedial education, rules, or discipline. As a frame of thought or cultural trait, the organization would seek to know more about the common-sense reasoning, such as the reasoning behind not seeking consultation or what made the actions appear correct. Common sense drives behaviors more rigorously than religion, philosophy, or science (11).

\section{Common sense and tacit knowledge}

Science has a formal, logical, and calculative structure. However, there is also a significant amount of tacit knowledge of those things that can be known only through living the collective life of science in specific expert communities. Such knowledge is not universal, immutable, articulated truths that can be knowable by anyone, or anything, anywhere (72). Tacit knowledge and common sense differ across domains (73) and are common only to the extent that two people share similar social and cultural experiences (12).

Common sense organizes knowledge in topological forms with relations for problem-solving, the nearness of relations increasing knowledge rather than logic operators. The person deals with a concrete situation on their terms. This differs from the academic approach of forming knowledge into logical categories (12). "Collective tacit knowledge" is more ontological, having to do with its location in the social collectivity (72), a topological structure.

Common sense treats as facts value judgments related to the socio-cultural environment (72). One difficulty of working with common sense knowledge is the association with identity and self-image. When questioned for a source, the person answers, "I just know it" (12). It was "common knowledge" through the 1990s that "rebound croup" following aerosolized adrenaline led physicians to withhold adrenaline to avoid hospital admission. This belief emerged from the original study using racemic epinephrine for croup, "symptoms often recurred within two hours, suggesting that this form of treatment should not be used in the emergency room and the patient then sent home" (74). Researchers continue to address this belief (75). The author (DvS) queried residents on whether it was rebound from the use of adrenaline or was it the fact that adrenaline lasts 0-45 minutes and it had ceased working. Several residents, insisting on the concept of rebound, commonly presented 3-5 critical and emergency care textbooks describing rebound croup and the use of adrenaline as an indication for hospitalization. No one believed in rebound headache after administration of one dose of ibuprofen.

Thus the many armed with prejudice and the few armed with logic fight an eternal battle, the logician charging the physical world with unintelligibility and the man of common sense charging the logical world with abstractness and unreality. The former view is the more profound since association by similarity is the more elementary and gives constancy to meanings. At the same time, the latter view is the more practical since association by contiguity alone informs the mind about the mechanical sequence of its own experience. Neither principle can be dispensed with, and each errs only in denouncing the other and wishing to be omnivorous, as if on the one hand logic could make anybody understand the history of events and the conjunction of objects, or on the other hand as if cognitive and moral processes could have any other terms than constant and ideal natures.

George Santayana (49)

Tacit knowledge is both somatic and cognitive. "Somatic knowledge," a form of biological knowledge like riding a bicycle, is difficult to describe but easy to demonstrate, therefore provides little contribution to Artificial Intelligence (72). Motor cognition is missed as combined somatic and cognitive knowledge, enhancing thinking while acting (45). Motor cognition describes how we adjust our actions to changing situations and how we learn through physical actions (76). Motor cognition, then, contributes to somatic common sense when we are actively engaged with an uncertain situation.

\section{"Motor cognition is missed as combined somatic and cognitive knowledge, enhancing thinking while acting (45). Motor cognition describes how we adjust our actions to changing situations and how we learn through physical actions (76)."}

\section{Misuse of common sense}

We must distinguish practical, common-sense problem solving used in the high-risk environment from common (sense) knowledge and other forms of "common sense" problem-solving. The phrase "common sense" often precedes a statement intended for acceptance without question. However, the characteristics of common sense form an inherent vice, the rapid connections from topological connections that effectively process large amounts of information can lead the inexperienced to believe they have gained insightful truths.

One difficulty working with common sense knowledge is the association with identity and self-image. When questioned for a source, the person answers, "I just know it" (12), and the inquiry ends. Others become offended when suggestions are not acted upon and less than cooperative in the engagement. The identity of individuals using practical common sense lies in the reduction of consequences 
Misused common sense supports prejudice and bias. Common sense treats as facts value judgments related to the socio-cultural environment (72). Improper analogies, misidentification of traits as useful, or focus on a few or minor traits can mislead and support bias and prejudice. Individuals using practical common sense can have biases and prejudices independent of common sense but will more likely dismiss them with the severity of consequences.

Facts that conform with a known rule or order become more convincing and more supportive of the rule or order. The individual believes the event is explained and is over or follows current knowledge (50). Contributing to the negative reputation of practical common is the priority of the actual world over scientific concepts and models (11).

\section{"Science translates connections within} topological space into hierarchies in Euclidean space (67) with loss of relations and information (50). Common

\section{sense derives its power and speed from topological connections."}

Science translates connections within topological space into hierarchies in Euclidean space (67) with loss of relations and information (50). Common sense derives its power and speed from topological connections.

Common sense can make it easy to subsume the event or results in preconceived patterns making our bias appear to result from deductive reasoning. It is not entirely invented, and it seems like part of what we know, but it is conformance to "vague self-knowledge" or a habitual way of thinking. It becomes obvious solely from persuasive words or lines of authority (50)

Obviousness and common agreement can assure us of being right to make "common-sense explanations so eminently satisfactory, as well as dangerous." The danger arises from using true knowledge without question, assuming the truth of analogies, leading to the misapplication of true knowledge (50). Practical common sense constantly questions the consequences. It is never over.

\section{Conclusion}

People develop their common sense from experience and modeling others while in their youth. What we have described is the practical common sense from lessons learned in blood. The consequences are real; they are deadly. Yes, the individual thinks rapidly, but it is speed from smoothness and intuition. What appears obvious is the rapid recognition of nuance in covert, compensated states. Nevertheless, the individual also ponders the situation and action. The judgment of irrelevancy is one of the more significant and dangerous; learning that irrelevant information was relevant always comes too late. This is practical common sense at the moment

Common sense also forms the basis of science, the initiator of scientific inquiry. Practical common-sense problem solving contributes to the refinement of scientific knowledge and extension of science into new realms. The British anthropologist Siegfried Frederick Nadel enlisted in the Sudan Defence Force early in 1941, later transferring to the British Army, East African Command, with service on the Eritrean-Ethiopian border and in charge of a frontier post (77). Nadel applied "psychological explanations" to anthropology in an effort to better understand the "why." $\mathrm{He}$ concludes his chapter "Explanation and Common Sense" with this paragraph (50):

"In a sense, we pile one regularity upon one we assume to be working behind it. The 'need' of both, a summary of the observed regular mode of behaviour and a machine invented to account for it. This is, of course, a circular argument, but the circularity as such is harmless - it is typical of many analytical (i.e., nondeductive) explanations. It is misleading only if it is taken for a deductive explanation, as though we had independent knowledge of the particular 'machine' and its efficiency, and not merely identified (not invented) its presence in an analogous situation. As we shall see, the confusion between invention and identification by analogy is often difficult to avoid; and even the exact sciences make use of such models of machines - and thus admit common sense; for a machine behind the observed regularity of phenomena is the most complete answer to the promptings of common sense. The motions of a round-about are puzzling only until we lift the trapdoor and discover a well-known engine driving the thing. Common sense always urges us to look for such trapdoors."

"Common sense also forms the basis of science, the initiator of scientific inquiry. Practical common-sense problem solving contributes to the refinement of scientific knowledge and extension of science into new realms."

\section{References}

1. Sternberg RJ, Wagner RK, Williams WM, Horvath JA. Testing common sense. American Psychologist. 1995;50(11):91227. doi: 10.1037/0003-066x.50.11.912.

2. Haas J. A study of high steel ironworkers' reactions to fear and danger. Sociology of work and occupations. 1977;4(2):14770.

3. Fitzpatrick JS. Adapting to danger: A participant observation study of an underground mine. Sociology of Work and Occupations. 1980;7(2):131-58.

4. van Stralen D, Mercer TA. The Nature of Neonatal Experience during Pandemic COVID-19. Neonatology Today. 2021;16(3):87-97. doi: 10.51362/neonatology.today/202131638797.

5. Thorvaldsen T. The importance of common sense: How Norwegian coastal fishermen deal with occupational risk. Marine Policy. 2013;42:85-90. doi: 10.1016/j.marpol.2013.02.007.

6. James $W$. The Present Dilemma in Philosophy. In: James $W$, editor. Pragmatism: A new name for some old ways of thinkin. New York, NY: Longman Green and Co 1907. p. 3-40.

\section{Readers can also follow} NEONATOLOGY TODAY via our Twitter Feed @NEOTODAY 
7. Dewey J. The pattern of inquiry Logic: The Theory of Inquiry. New York, NY: Holt, Rinehart and Winston; 1938. p. 101-19.

8. Jovchelovitch S. The Rehabilitation of Common Sense: Social Representations, Science and Cognitive Polyphasia. Journal for the Theory of Social Behaviour. 2008;38(4):43148. doi: 10.1111/j.1468-5914.2008.00378.x.

9. Parnas J, Bovet P. Autism in schizophrenia revisited. Comprehensive psychiatry. 1991;32(1):7-21.

10. Fletcher GJ. Psychology and common sense. American Psychologist. 1984;39(3):203-13. doi: 10.1037/0003066x.39.3.203.

11. Geertz C. Common Sense as a Cultural System. The Antioch Review. 1975;33(1). doi: 10.2307/4637616.

12. Watts DJ. Common sense and sociological explanations. AJS. 2014;120(2):313-51. Epub 2015/03/27. doi: 10.1086/678271. PubMed PMID: 25811066.

13. Taylor CC. Sociology and Common Sense. American Sociological Review. 1947;12(1). doi: 10.2307/2086483.

14. Furnham A. Social psychology as common sense. Bulletin of the British Psychological Society 1983;36:105-9.

15. McCarthy J. Artificial intelligence, logic and formalizing common sense. In: R.H. T, editor. Philosophical logic and artificial intelligence. Berlin, Germany: Springer, Dordrecht; 1989. p. 161-90.

16. Carbonell JG, Minton S. Metaphor and common-sense reasoning. Arlington, VA: Carnegie-Mellon University, 1983.

17. McCarthy J. Ascribing Mental Qualities to Machines. Palo Alto, CA: Stanford University, Stanford Artificial Intelligence Laboratory CSD; 1979 STAN-CS-79-725.

18. Minsky M. How to Teach a Robot. Naval Research Reviews 1972;25(12):1-13.

19. Stanghellini G. Vulnerability to schizophrenia and lack of common sense. Schizophr Bull. 2000;26(4):775-87. Epub 2000/11/22. doi: 10.1093/oxfordjournals.schbul.a033493. PubMed PMID: 11087011.

20. Horton $R$. Tradition and modernity revisited. In: Hollis M, Lukes S, editors. Rationality and Relativis. Oxford, U.K.: Basil Blackwell; 1982. p. 201-60.

21. Flick U. Everyday knowledge in social psychology. In: Flick $U$, editor. The Psychology of the Social. Cambridge, UK: Cambridge University Press; 1998. p. 41-59.

22. Damasio AR. Looking for Spinoza: Joy, sorrow, and the feeling brain. Orlando, FL: Harcourt, Inc.; 2003.

23. van Stralen D. Pragmatic High-Reliability Organization (HRO) During Pandemic COVID-19. Neonatology Today. 2020;15(4):3-9.

24. van Stralen D, Mercer TA. High-Reliability Organizing (HRO) in the COVID-19 Liminal Zone: Characteristics of Workers and Local Leaders. Neonatology Today. 2021;16(4):90-101. doi: 10.51362/neonatology.today/2021416490101.

25. van Stralen D, Gambino W. Error as a Faulty Failure Signal. Neonatology Today. 2020;15(9):114-7. doi: 10.51362/neonatology.today/20209159114117.

26. van Stralen D, Mercer TA. Pragmatic High-Reliability Organizations (HRO) Modulate the Functions of Stress and Fear Behaviors During Pandemic COVID-19: The Stress-FearThreat Cascade. Neonatology Today. 2020;15(10):126-34. doi: 10.51362/neonatology.today/2020101510126134.

27. van Stralen D, McKay S, Mercer TA. Pragmatic Leadership Practices in Dangerous Contexts: High-Reliability Organizations (HRO) for Pandemic COVID-19. Neonatology
Today. 2020;15(8):109-17. doi: 10.51362/neonatology.today/20208158109117.

28. Eisenberg J, Lysouvakon P, Hageman J. Management of a Symptomatic COVID-19 Positive Pregnant Woman and $\mathrm{Her}$ Newborn Infant. Neonatology Today. 2020;15(8):105-7. doi: 10.51362/neonatology.today/20208158105107.

29. van Stralen D, Lysouvakon $P$, Hageman JR, Eisenberg $J$, Goldstein M. Letter to the Editor. Neonatology Today. 2020;15(9):121-3. doi: 10.51362/neonatology.today/20209159121123.

30. Simon HA. The structure of ill structured problems. Artificial Intelligence. 1973;4(3-4):181-201. doi: 10.1016/00043702(73)90011-8.

31. Weick KE. The collapse of sensemaking in organizations: The Mann Gulch disaster. Administrative science quarterly. 1993;38(4):628-52.

32. van Stralen D, McKay S, Mercer T. Flight Decks and Isolettes: High-Reliability Organizing (HRO) as Pragmatic Leadership Principles during Pandemic COVID-19. Neonatology Today. 2020;15(7):113-21. doi: 10.51362/neonatology.today/20207157113121.

33. Arnold III AV. Strategic visioning: What it is and how it's done. Carlisle Barracks, PA: United States Army War College, 1991.

34. Magee RR. Strategic leadership primer. Carlisle Barracks, PA: United States Army War College, 1998.

35. Szakolczai A. Liminality and experience: Structuring transitory situations and transformative events. International Political Anthropology. 2009;2(1):141-72.

36. Weick KE. Sensemaking in organizations. Dickens $G$, editor. Thousand Oaks, CA: Sage; 1995.

37. McCarthy J, Hayes PJ. Some Philosophical Problems from the Standpoint of Artificial Intelligence. In: Meltzer B, Michie $D$, editors. Machine Intelligence 4. Edinburgh, Scotland: Edinburgh University Press; 1969. p. 463--502.

38. Watts DJ. Everything is obvious *Once you know the answer: How common sense fails. London, UK: Atlantic Books;

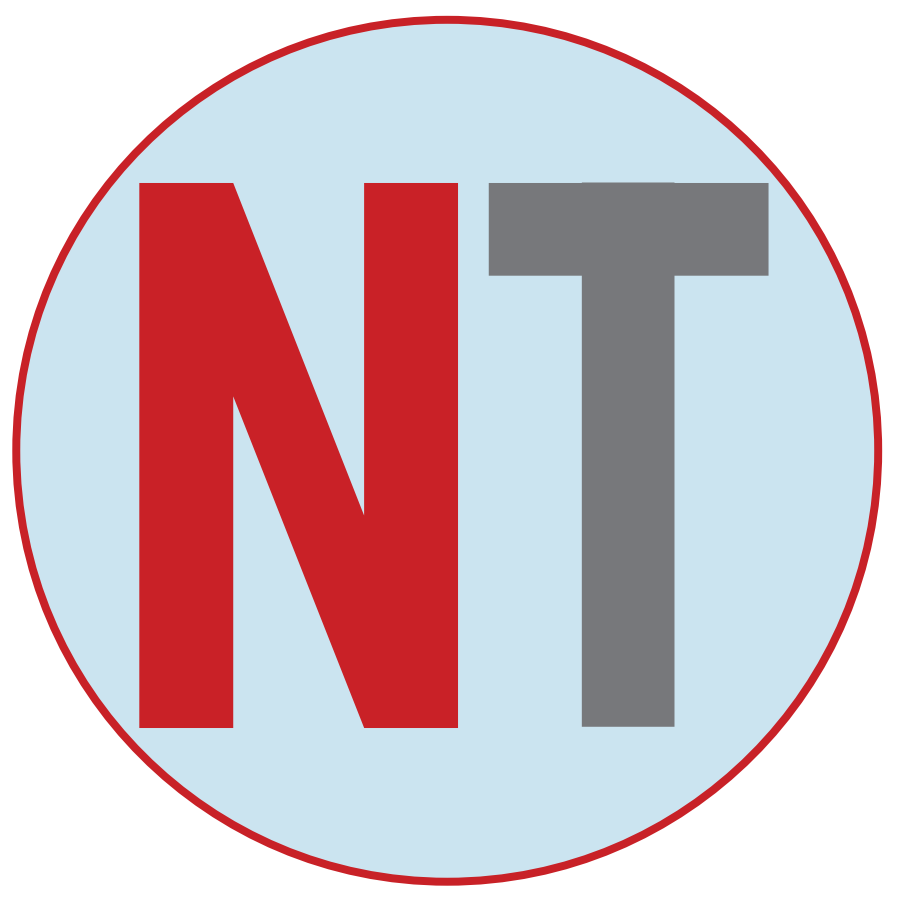


2011.

39. James W. Pragmatism and Common Sense Pragmatism and four essays from the meaning of truth. Cleveland, $\mathrm{OH}$ : World Publishing Company; 1909. p. 111-30.

40. Mishara AL. On Wolfgang Blankenburg, Common Sense, and Schizophrenia. Philosophy, Psychiatry, \&amp; Psychology. 2001;8(4):317-22. doi: 10.1353/ppp.2002.0021.

41. Fitzgerald M. Overlap between autism and schizophrenia: history and current status. Advances in Mental Health and Intellectual Disabilities. 2014;8(1):15-23. doi: 10.1108/amhid-09-2013-0058.

42. van Stralen D, Mercer TA. Pandemic COVID-19, the HighReliability Organization (HRO), and the Ecology of Fear. Neonatology Today. 2020;15(12):129-38. doi: 10.51362/ neonatology.today/2020121512129138.

43. Westrum R. A typology of organisational cultures. Quality and Safety in Health Care. 2004;13(suppl_2):ii22-ii7. doi: 10.1136/qshc.2003.009522.

44. Weick KE, Sutcliffe KM. Managing the Unexpected: Assuring High Performance in an Age of Complexity. Quinn RE, editor. San Francisco, CA: Jossey-Bass; 2001.

45. Weick KE. Enacted sensemaking in crisis situations Journal of management studies. 1988;25(4):305-17.

46. Simon HA, Newell A. Heuristic Problem Solving: The Next Advance in Operations Research. Operations Research. 1958;6(1):1-10. doi: 10.1287/opre.6.1.1.

47. van Stralen D, Inozu B, Byrum S. High Reliability for a Highly Unreliable World: Preparing for Code Blue through Daily Operations in Healthcare. North Charleston, SC: CreatSpace Publishing; 2017.

48. Hickman LA. Pragmatism as post-postmodernism: Lessons from John Dewey. New York, NY: Fordham University Press; 2007.

49. Santayana G. Concretions in Discourse and in Existence Reason in common sense. The Life of Reason or the Phases of Human Progress I. London, UK: Archibald Constable \& Co.; 1910. p. 161-83.

50. Nadel SF. The Foundations of Social Anthropology. London, UK: Routledge; 1951.

51. Hunter $W$. On the Uncertainty of the Signs of Murder in the case of Bastard Children. In: Cummin W, editor. The Proofs of Infanticide Considered: Including Dr Hunter's Tract on Child Murder, with Illustrative Notes; and a Summary of the Present State of Medico-legal Knowledge on that Subject. London, UK: Longman, Rees, Orme, Brown, Green, and Longman; 1783. p. 2-20.

52. Terry H. An Account of Two Stillborn Children, Restored to Life a Long Time after Birth. Edinburgh medical and surgical journal. 1818;14(54):183-6.

53. Wilson J. Cases of Asphyxia. Glasgow medical journal 1829;2(7):237-40.

54. Culbreth RE, Gardenhire DS. Manual bag valve mask ventilation performance among respiratory therapists. Heart \& Lung. 2021;50(3):471-5.

55. von Goedecke A, Wagner-Berger HG, Stadlbauer KH, Krismer AC, Jakubaszko J, Bratschke $C$, et al. Effects of decreasing peak flow rate on stomach inflation during bagvalve-mask ventilation. Resuscitation. 2004;63(2):131-6.

56. van Stralen D, Westmoreland T. Management of a Mechanical Ventilator Using Patient Calm as an Endpoint. Special Operations Medical Association Scientific Assembly (SOM-
SA); December 8-11, 2014; Tampa, Florida: Special Operations Medical Association (SOMA); 2014.

57. Smillie S. The Last Sea Nomads: Inside the disappearing world of the Moken. London, UK: The Guardian; 2014.

58. Bhaumik S. Tsunami Folklore 'Saved Islanders'. UK: BBC News; 2005.

59. Minsky M. A framework for representing knowledge. In: Winston PH, editor. The Psychology of Computer Vision. New York, NY: 211-277; 1975.

60. Nelson B, Whitford TJ, Lavoie S, Sass LA. What are the neurocognitive correlates of basic self-disturbance in schizophrenia? Integrating phenomenology and neurocognition: Part 2 (aberrant salience). Schizophr Res. 2014;152(1):207. Epub 2013/07/19. doi: 10.1016/j.schres.2013.06.033. PubMed PMID: 23863772.

61. Sommerville JA, Decety J. Weaving the fabric of social interaction: articulating developmental psychology and cognitive neuroscience in the domain of motor cognition. Psychon Bull Rev. 2006;13(2):179-200. Epub 2006/08/09. doi: 10.3758/ bf03193831. PubMed PMID: 16892982.

62. Davis E, Marcus G. Commonsense reasoning and commonsense knowledge in artificial intelligence. Communications of the ACM. 2015;58(9):92-103. doi: 10.1145/2701413.

63. McCarthy J. Some expert systems need common sense. Ann N Y Acad Sci. 1984;426:129-37. Epub 1984/01/01. doi: 10.1111/j.1749-6632.1984.tb16516.x. PubMed PMID: 6391331.

64. Naudin J, Azorin J, Mishara AL, Wiggins OP, Schwartz M. Schizophrenia and common sense: study of 3 single cases. Psychopathology. 2000;33(5):275-82. Epub 2000/08/31. doi: 10.1159/000029158. PubMed PMID: 10965286.

65. van Stralen $D$, Mercer TA. High-Reliability Organizing (HRO) and Abrupt Change from COVID 19: Failure of Scientific Rationality and Classical Logic. Neonatology Today. 2021;16(6): 97-109. doi: https://doi.org/10.51362/neonatology.today/2021616697109

66. Clement CA, Gentner D. Systematicity as a selection constraint in analogical mapping. Cognitive science. 1991;15(1):89-132.

67. Asher $N$, Vieu L, editors. Toward a geometry of common sense: A semantics and a complete axiomatization of mereotopology. IJCAI (1); 1995: Citeseer.

68. Dennett DC. Cognitive Wheels: The frame problem of Al. In: Hookway C, editor. Minds, Machines and Evolution. Cambridge, UK: Cambridge University Press; 1984. p. 129-51.

69. Bickhard MH. Why Children Don't Have to Solve the Frame Problems: Cognitive Representations Are Not Encodings. Developmental Review. 2001;21(2):224-62. doi: 10.1006/ drev.2000.0521.

70. Fletcher GJO. Psychology and Common Sense. IEEE Engineering Management Review. 1986;14(4):30-40. doi: 10.1109/emr.1986.4306241.

71. Snir I. Making sense in education: Deleuze on thinking against common sense. Educational Philosophy and Theory. 2017;50(3):299-311. doi: 10.1080/00131857.2017.1344537.

72. Collins H. Bicycling on the Moon: Collective Tacit Knowledge and Somatic-limit Tacit Knowledge. Organization Studies. 2006;28(2):257-62. doi: 10.1177/0170840606073759.

73. Schank RC, Abelson RP. Scripts, Plans, Goals, and Understanding: An Inquiry into Human Knowledge. Hillsdale, NJ: Erlbaum; 1977. 
74. Taussig LM, Castro O, Beaudry PH, Fox WW, Bureau M. Treatment of laryngotracheobronchitis (croup): use of intermittent positive-pressure breathing and racemic epinephrine. American Journal of Diseases of Children. 1975;129(7):790-3.

75. Bagwell T, Hollingsworth A, Thompson T, Abramo T, Huckabee $M$, Chang $D$, et al. Management of croup in the emergency department: the role of multidose nebulized epinephrine. Pediatric emergency care. 2020;36(7):e387-e92.

76. van Stralen D, Mercer T. High Reliability Organizing (HRO) is the Extension of Neonatology during Pandemic COVID-19. Neonatology Today. 2021;16(5):97-109. doi: 10.51362/neonatology.today/2021516597109.

77. Firth R. Siegfried Frederick Nadel, 1903-1956. American Anthropologist 1957;59:117-24.

Disclosures: The authors have no relevant disclosures

NT
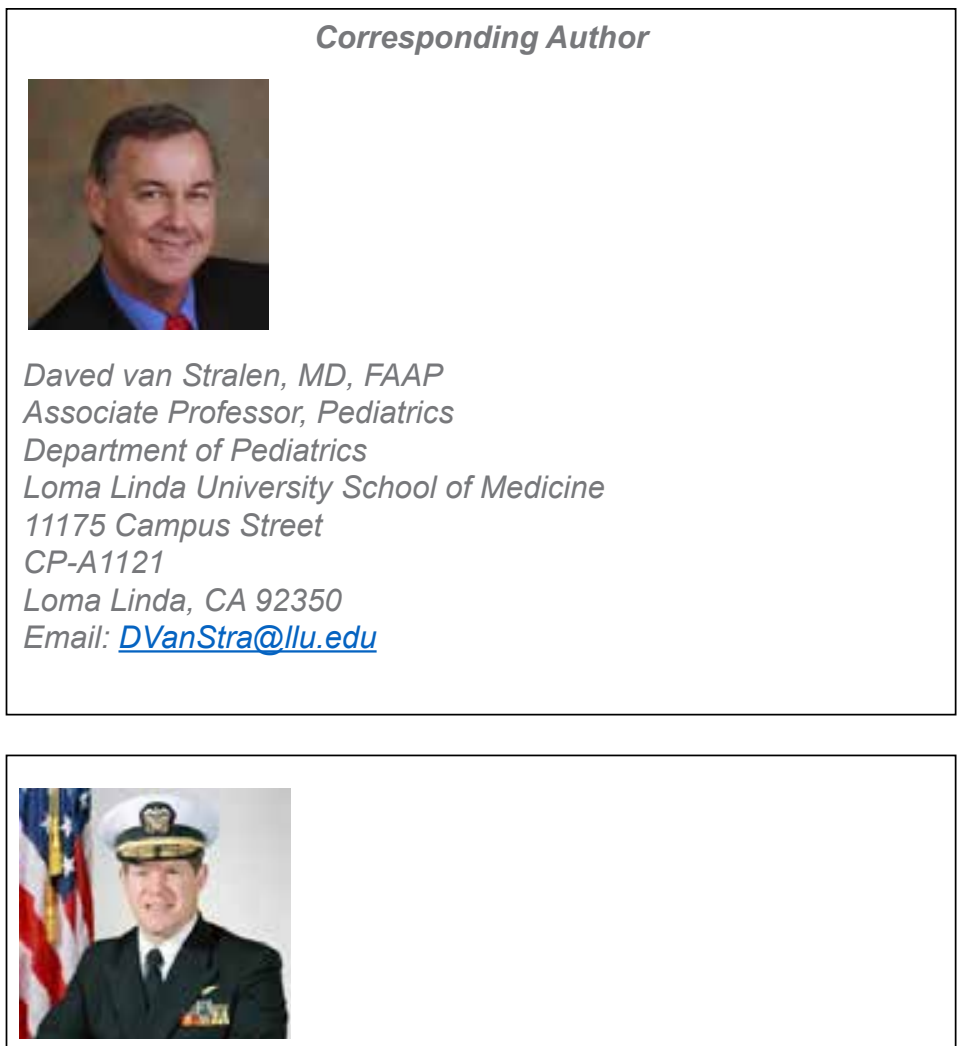

Thomas A. Mercer

Rear Admiral

United States Navy (Retired)

\section{Acknowledgments}

Karl Weick, Rensis Likert Distinguished University Professor of Organizational Behavior and Psychology, Emeritus, University of Michigan

Errol van Stralen, Ancora Education

William Gambino, CIV, DoD

Sean D. McKay, Element Rescue, LLC

Laura Elliott, Children's Subacute Center, Community Hospital of San Bernardin

William J. Corr, formerly with the Los Angeles City Fire Department, now deceased
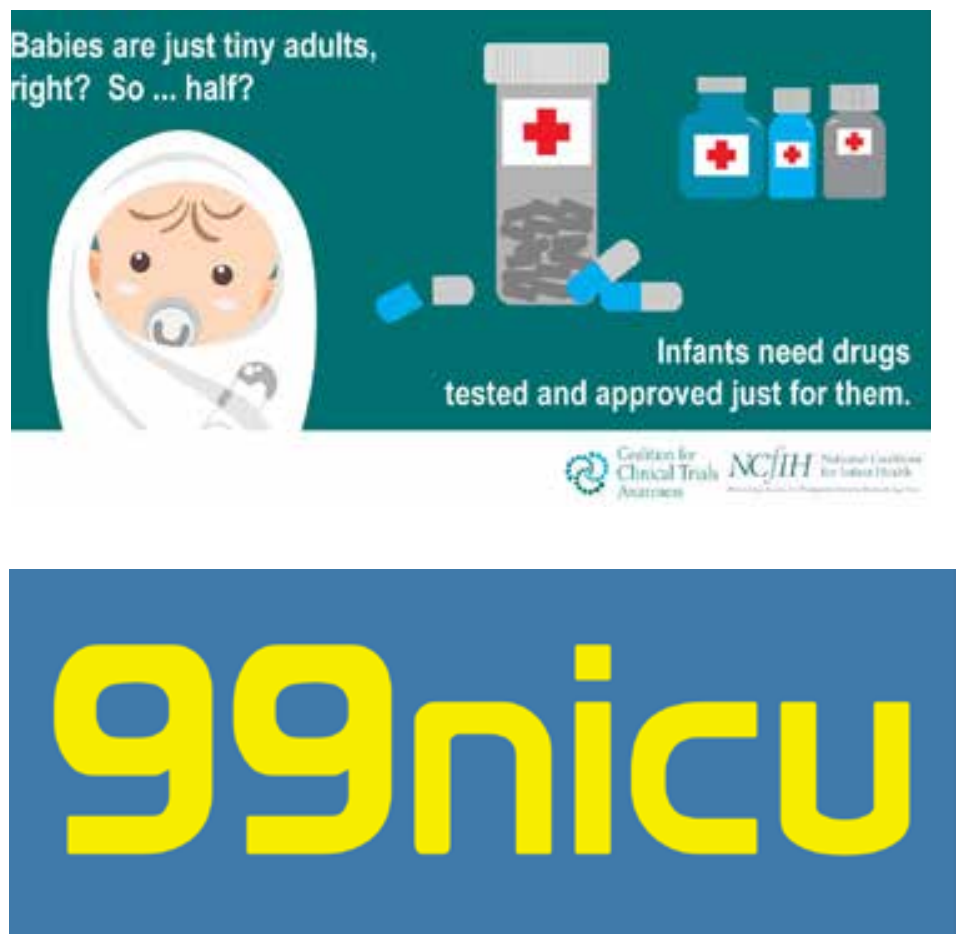

$$
\begin{aligned}
& \text { Readers can also follow } \\
& \text { NEONATOLOGY } \\
& \text { via our Twitter Feed } \\
& \text { @NEOTO }
\end{aligned}
$$

NEONATOLOGY TODAY is interested in publishing manuscripts from Neonatologists, Fellows, NNPs and those involved in caring for neonates on case studies, research results, hospital news, meeting announcements, and other pertinent topics.

Please submit your manuscript to: LomaLindaPublishingCompany@gmail.com 